OPEN ACCESS

Edited by:

Song Sun,

China Academy of Engineering

Physics, China

Reviewed by:

Kien Voon Kong,

National Taiwan University, Taiwan

Taiping Zhang,

China Academy of Engineering

Physics, China

${ }^{*}$ Correspondence:

Xiaosheng Zhang

zhangxs@uestc.edu.cn

Specialty section:

This article was submitted to

Optics and Photonics,

a section of the journal

Frontiers in Physics

Received: 05 April 2021

Accepted: 13 April 2021

Published: 20 May 2021

Citation:

Gong T, Guan F, Wei Z, Huang W and Zhang X (2021) Tunable Magnetic

Fano Resonances on Au Nanosphere

Dimer-Dielectric-Gold Film

Sandwiched Structure.

Front. Phys. 9:691027.

doi: 10.3389/fphy.2021.691027

\section{Tunable Magnetic Fano Resonances on Au Nanosphere Dimer-Dielectric-Gold Film Sandwiched Structure}

\author{
Tianxun Gong, Fang Guan, Zhenjiang Wei, Wen Huang and Xiaosheng Zhang* \\ State Key Laboratory of Electronic Thin Films and Integrated Devices, School of Electronic Science and Engineering (National \\ Exemplary School of Microelectronics), University of Electronic Science and Technology of China, Chengdu, China
}

The Fano resonance demonstrates excellent performance due to its narrow asymmetrical spectral line shape, and its sensitivity to structure and material parameter changes. Compared to conventional Fano resonances, the Fano resonance generated by the magnetic dipole is more advantageous because of its high absorption and low loss. In this study, we propose an Au nanosphere dimer-dielectric-gold film sandwiched structure that supports the magnetic Fano resonance mode. And the Fano resonance can be efficiently tuned from visible to near-infrared wavelength by changing the size of the gold nanospheres and the thickness of the dielectric layer. Different from the single gold nanosphere-dielectric-film structure, the results suggest that the proposed structure is sensitive to the polarization direction of the excitation light. Considering the above characteristics, the proposed structure can be applied in multi-band sensing, surface-enhanced Raman spectroscopy, and dynamically adjustable optical switches.

Keywords: Fano resonance, magnetic mode, surface plasmons, Au nanosphere dimer-dielectric-gold film, radiation loss

\section{INTRODUCTION}

Surface plasmon is generated by collective oscillations of photons and electrons [1,2]. Due to the large radiation loss in the visible light band, surface plasmon resonance has a wide Lorentzian profile resonance peak, which limits its performance [3]. In contrast, Fano resonance originated from the constructive and destructive interference of light and dark modes can effectively suppress the radiation loss and generate strong surface electromagnetic enhancement [4-8]. Furthermore, Fano resonance is drawing intense interest due to its high sensitivity to geometric parameters and the refractive index changes [7, 9-13]. Therefore, Fano resonance can be used in many applications, such as surface-enhanced Raman spectroscopy, surface-enhanced fluorescence, surface plasmon photon chips, resonators, nano-antennas, couplers, biosensors, and optical filtering [14-23].

Most of the researchers who studied Fano resonance focused on the electric effect reflected in the coupling between the electric dipole and the higher-order electric mode [8, 18, 24-26]. Only in recent years, Fano resonance generated by the magnetic mode is being studied [27-29]. However, the mechanisms of magnetic dipole mode have been discussed in previous studies [30, 31]. The magnetic modes can be excited by the sandwiched structure connecting metal layers with a dielectric layer, which is equivalent to a capacitor model. While the two layers of the metal function as two electrodes, opposite charge distributions are generated on the two layers so that a circular 
displacement current is formed between the metal layers. In such a case, the dipole moment is reduced, which effectively inhibits the loss of the system. According to the present research, organic molecules and two-dimensional materials such as PEG, graphene, and $\mathrm{MgF}_{2}$, function as dielectric layers [14, 32, 33]. However, the mechanism of effective tuning of the magnetic Fano resonance mode is not studied in depth. In this study, we propose a sandwiched structure composed of an Au dimer and an Au film linked by a dielectric molecule layer, which supports magnetic Fano resonance that can be tuned by the radius of the nanosphere and the thickness of the dielectric layer. Moreover, the proposed structure is sensitive to the polarization direction of the excitation light. Owing to its huge field enhancement brought about by the generation of magnetic mode, the proposed structure has potential applications in improving the sensitivity of multi-band sensing, surface-enhanced Raman spectroscopy, and dynamically adjustable optical switches.

\section{STRUCTURE AND SIMULATION METHOD}

The structure was calculated by the finite element method (FEM). A three-dimensional model was developed for the calculation, in which the top and bottom boundaries were set as input port and output port, respectively. For the input port, a normal incident excitation wave with electric field components along the $x$ and/or $y$ direction was adopted during the study. A schematic illustration of the structure is shown in Figure 1. As shown in Figure $1 \mathrm{~A}, R_{1}$ and $R_{2}$ are the radii of two $\mathrm{Au}$ nanospheres, respectively. The distance between the two Au nanospheres is fixed at $5 \mathrm{~nm}$. The Au film thickness $(D)$ was fixed at $200 \mathrm{~nm}$. The refractive index of the dielectric layer is 1.469 . The thickness of the dielectric layer between the $\mathrm{Au}$ nanospheres (radii $R_{1}$ and $R_{2}$ ) and the gold film are $t_{1}$ and $t_{2}$, respectively. The calculated absorption cross-section of the structure with $R_{1}$ and $R_{2}$ set at $100 \mathrm{~nm}$ and $t_{1}$ and $t_{2}$ set at $1 \mathrm{~nm}$ is shown in Figure 1B, in which the Fano resonance can be observed with two distinct peaks located at $700 \mathrm{~nm}$ (marked as "1") and $900 \mathrm{~nm}$ (marked as “2”).

\section{RESULTS AND DISCUSSION}

\section{The Mechanism of Fano Resonance}

To study the mechanism of Fano resonance for the structure, the distribution of the electric field, the magnetic field, and the surface charge corresponding to the structures with $R_{1}$ varying from 80 to $120 \mathrm{~nm}$ at the two absorption peaks marked as Peak 1 (near $700 \mathrm{~nm}$ ) and Peak 2 (near $900 \mathrm{~nm}$ ) are calculated, as shown in Figures $2 \mathrm{C}-\mathbf{L}$, respectively. The displacement current vector is indicated by the arrow with white color in the distribution of the electric field. As $R_{1}=R_{2}=100 \mathrm{~nm}$ and $t_{1}=t_{2}=$ $1 \mathrm{~nm}$, the distribution of the electric field, the magnetic field, and the surface charge at Peak 1 are shown in Figures 2Ei, ii, iii, respectively. Two electric current loops formed by the displacement current vector between $\mathrm{Au}$ nanospheres with $\mathrm{Au}$ film can be observed in the electric field distribution (Figure 2E-i). The current loops induce the magnetic mode, which can be seen from the anti-symmetric charge distribution (Figure 2E-iii) of the Au nanosphere and the Au film [31]. On the other hand, the electric dipole mode is excited between two spheres, as the magnetic field hotspot between the two spheres is relatively weak (Figure 2E-ii). Therefore, the optical properties at Peak 1 are dominated by the magnetic dipole mode. For Peak 2, no current loop could be observed between the Au nanospheres and the gold film (Figure 2J-i), while the near-field electric intensity between the nanospheres is relatively strong. Meanwhile, from its surface charge distribution (Figure 2J-iii), it can be seen that the electric dipole is located between the two nanospheres. Hence, the optical properties at Peak 2 are

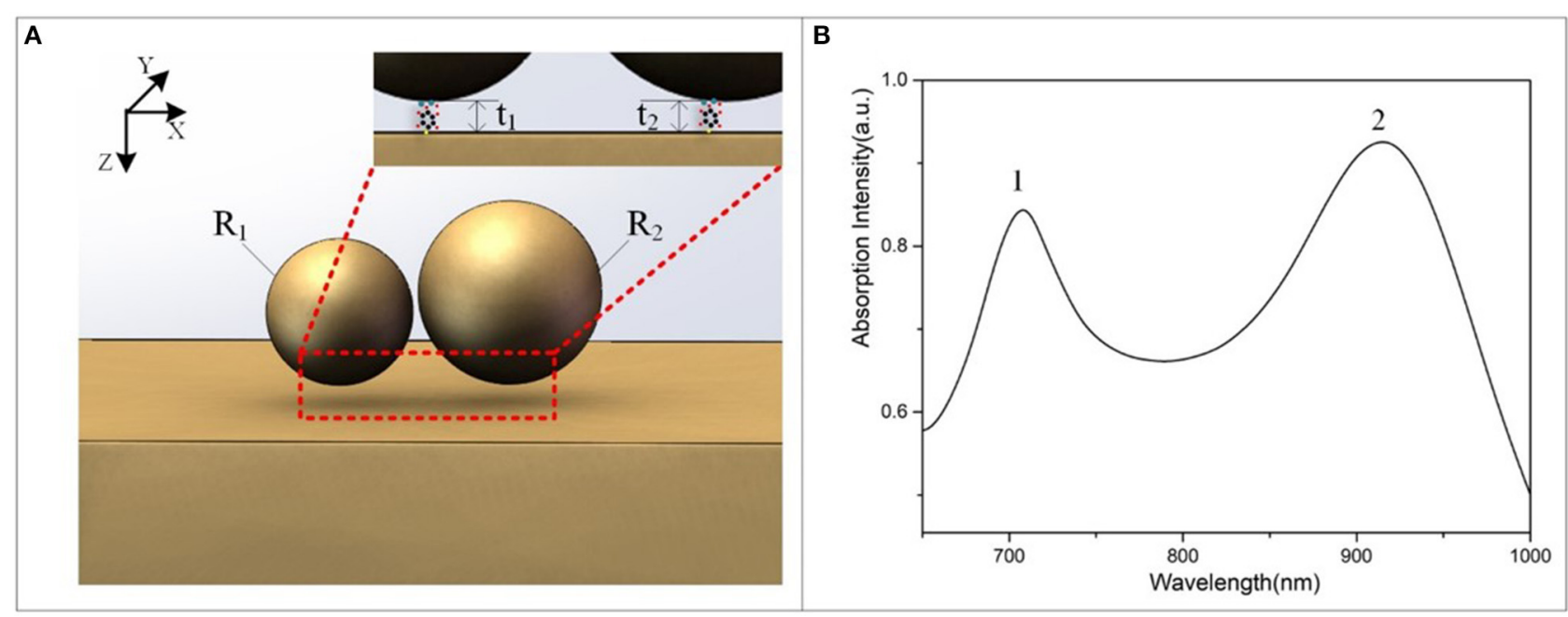

FIGURE 1 | (A) The schematic of Au nanosphere dimer-dielectric-Au film plasmonic nanostructures. The radii of two Au nanospheres are $R_{1}$ and $R_{2}$, respectively. The thickness of the dielectric layer under two Au nanospheres is $t_{1}$ and $t_{2}$, respectively. The Au film has a thickness of $D$. (B) The calculated absorption cross-section of the structure $\left(R_{1}=R_{2}=100 \mathrm{~nm}, t_{1}=t_{2}=1 \mathrm{~nm}, D=200 \mathrm{~nm}\right)$. 


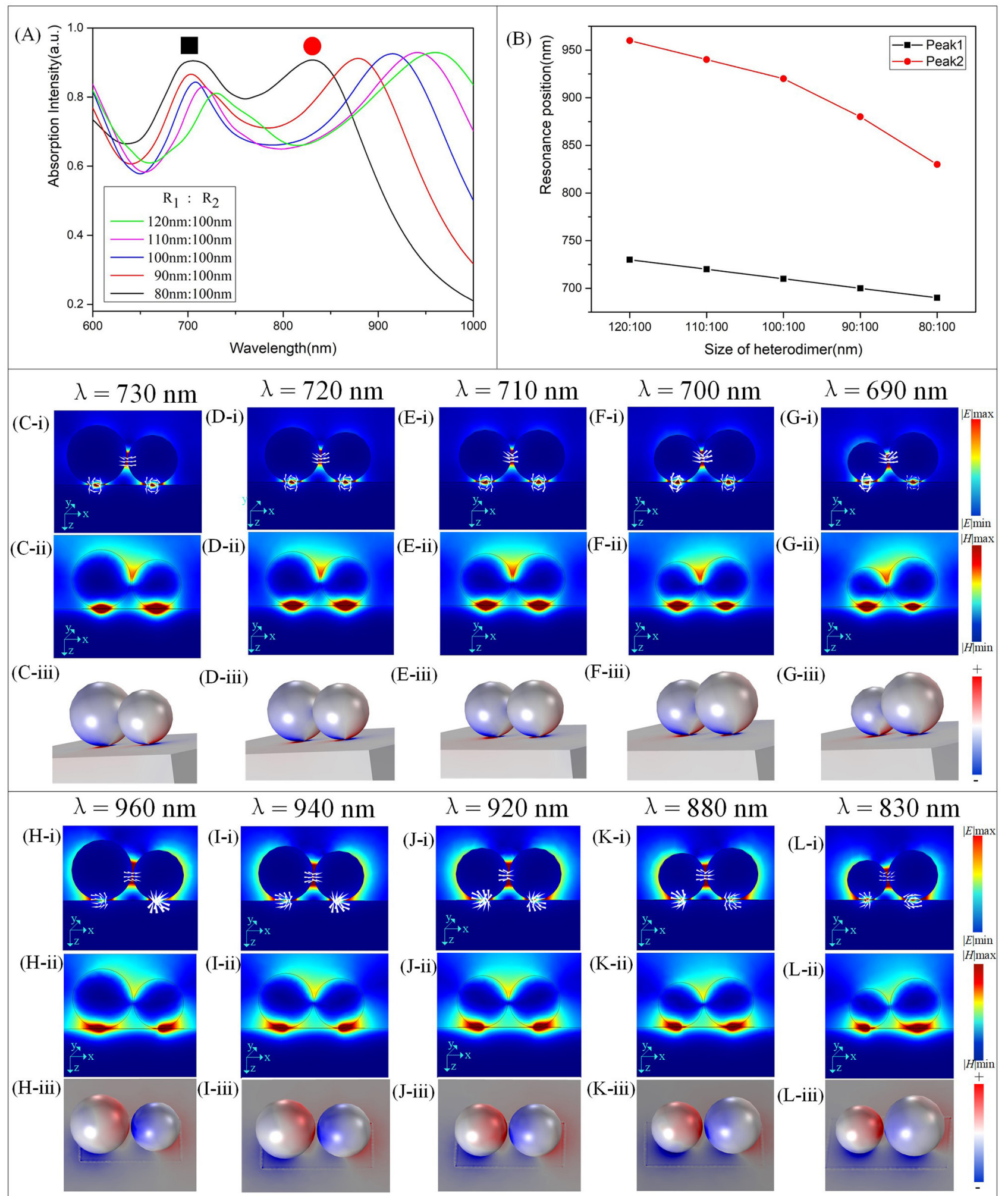

FIGURE 2 | (A) Absorption spectra of Au nanosphere dimer-dielectric-Au film nanostructures with $R_{1}$ varied from 120 to $80 \mathrm{~nm}$, while $R_{2}$ is fixed at $100 \mathrm{~nm}$. Peak 1 (black square) and Peak 2 (red circle) are marked in the absorption spectrum. (B) The resonance peaks as a function of the nanosphere dimer size. (C-G) Distribution of the electric field (i), the magnetic field (ii), and the surface charge (iii) at Peak 1 corresponding to the nanosphere dimer with $R_{1}$ from 120 to 80 nm. (H-L) Distribution of the electric field (i), the magnetic field (ii), and the surface charge (iii) at Peak 2 corresponding to the nanosphere dimer with $R_{1}$ from 120 to 80 nm. 


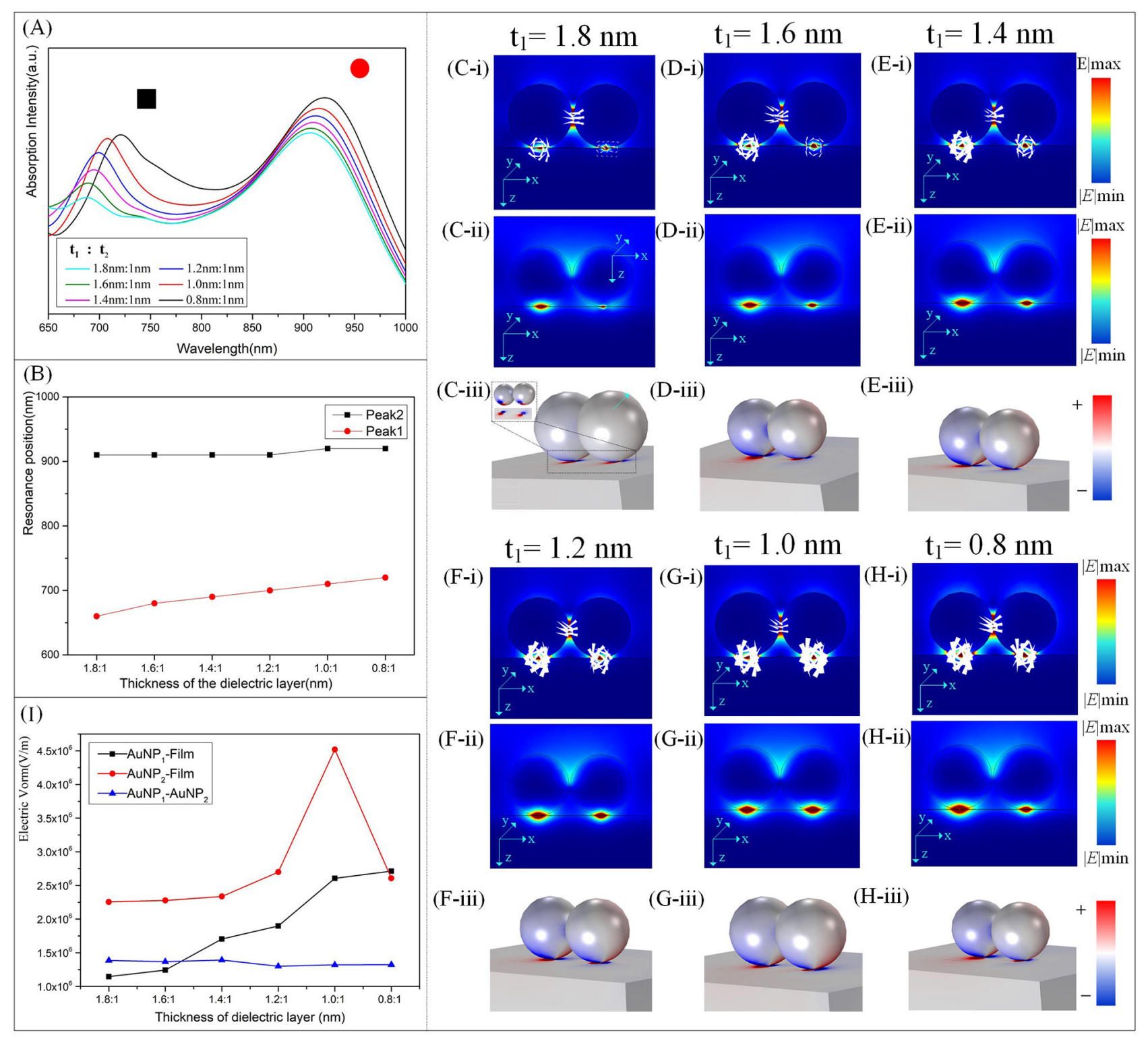

FIGURE 3 | (A) Absorption spectra of the Au nanosphere dimer-dielectric-Au film nanostructures with $t_{1}$ varied from 1.8 to $0.8 \mathrm{~nm}$, whereas $t_{2}$ is fixed at $1 \mathrm{~nm}$. (B) The resonance peaks as a function of the thickness of the dielectric layer. (C-H) Distribution of the electric field (i), the magnetic field (ii), and the surface charge (iii) at Peak 1 corresponding to nanosphere dimer with $t_{1}$ from 1.8 to $0.8 \mathrm{~nm}$. (I) Electric field enhancement of three hot spots at Peak 1 as a function of $t_{1}$.

dominated by the electric dipole mode, and the hotspot between $\mathrm{Au}$ nanospheres and $\mathrm{Au}$ film in the magnetic field distribution (Figure 2J-ii) is not extremely localized compared with that of Peak 1 (Figure 2E-ii). Therefore, the generation of the Fano resonance is due to the coupling of the two modes: the magnetic dipole mode as the dark mode with the smaller net dipole moment and the electric dipole mode as the bright mode.

\section{Effects of Nanosphere Radius}

The effects of the $R_{1}$ on Fano resonance are also studied. Figure $2 \mathrm{~A}$ shows the absorption spectrum of the structure with $R_{1}$ tuning from 80 to $120 \mathrm{~nm}$. As $R_{1}$ increases, in addition to a redshift of both peaks 1 and 2, a slight broadening of the Fano dip is also observed in the spectrum. As shown in Figure $\mathbf{2 B}$, the location of Peak 1 shifted from 690 to $730 \mathrm{~nm}$, and Peak 2 shifted from 830 to $960 \mathrm{~nm}$. Despite the changes in $R_{1}$, the magnetic dipole mode between the Au nanosphere dimer and the Au film dominates at Peak 1. There is an obvious current loop observed in the electric field distribution (Figures 2C-G-i), and the surface charge distribution is anti-phase symmetrical (Figures 2C-G-iii). The electric dipole mode dominates at Peak 2, which can be observed from Figures $\mathbf{2 H}-\mathbf{L}$. In the meantime, as the $R_{1}$ radius increases, the magnetic field intensity and electric dipole forces also increase, resulting in the enhancement of the near-field 
interaction in the system. As a result, the mode shifts to lower energies, and the Fano dip is broadened [34].

\section{Effects of the Dielectric Layer Thickness}

The influence of $t_{1}$ on Fano resonance is also studied. Figure 3A shows the absorption spectrum of the structure with $t_{1}$ from 0.8 to $1.8 \mathrm{~nm}$. With the decrease in the $t_{1}$, the shifts of the two resonance peak positions are shown in Figure 3B. There was an insignificant shift in the position of Peak 2, which was fixed around $920 \mathrm{~nm}$. As Peak 2 originated from the mutual coupling between the two Au nanospheres, the variation in the dielectric layer thickness has little effect on the relative positions of the two Au nanoparticles. The change in the coupling degree and the electric dipole moment was insignificant; therefore, the position of Peak 2 was almost unchanged. On the contrary, as $t_{1}$ decreased from 1.8 to $0.8 \mathrm{~nm}$, the position of Peak 1 (red) shifted from 660 to $720 \mathrm{~nm}$. Figures $3 \mathrm{C}-\mathbf{H}$ shows the distribution of electric field (i), magnetic field (ii), and surface charge (iii) when the dielectric layer thickness $t_{1}$ decreased from 1.8 to $0.8 \mathrm{~nm}$.
The magnetic dipole force between the Au nanospheres $R_{1}$ and the Au film connected by the dielectric layer with a thickness of $t_{1}$ continuously decreased, and the magnetic field hotspots became stronger. In addition, it was found that plasmon mode and intensity excited between the Au nanosphere $R_{2}$ and the $\mathrm{Au}$ film connected by the dielectric layer with a thickness of $t_{2}$ were also affected. When the thickness $t_{1}$ was $1.8 \mathrm{~nm}$, the magnetic quadrupole mode was excited between the Au nanosphere $R_{2}$ and the Au film, as shown in Figure 3C-iii. The inset is the detailed schematic diagram of the charge distribution at the junction. However, due to the low displacement current density, the localized near-field magnetic intensity becomes weak [29]. Apart from the case of $1.8 \mathrm{~nm}$, as $t_{1}$ decreases from 1.6 to $0.8 \mathrm{~nm}$, the magnetic dipole mode is stimulated at the junction of the nanosphere with a radius of $R_{2}$ and the film (with a thickness of $t_{1}$ ), as shown in Figures 3D-H-i. The electric and magnetic intensities reached the highest when $t_{1}$ was $1 \mathrm{~nm}$ and gradually decreased with an increase in $t_{1}$, as shown in Figure 3I. It was also observed from the near-field electric intensity at the three
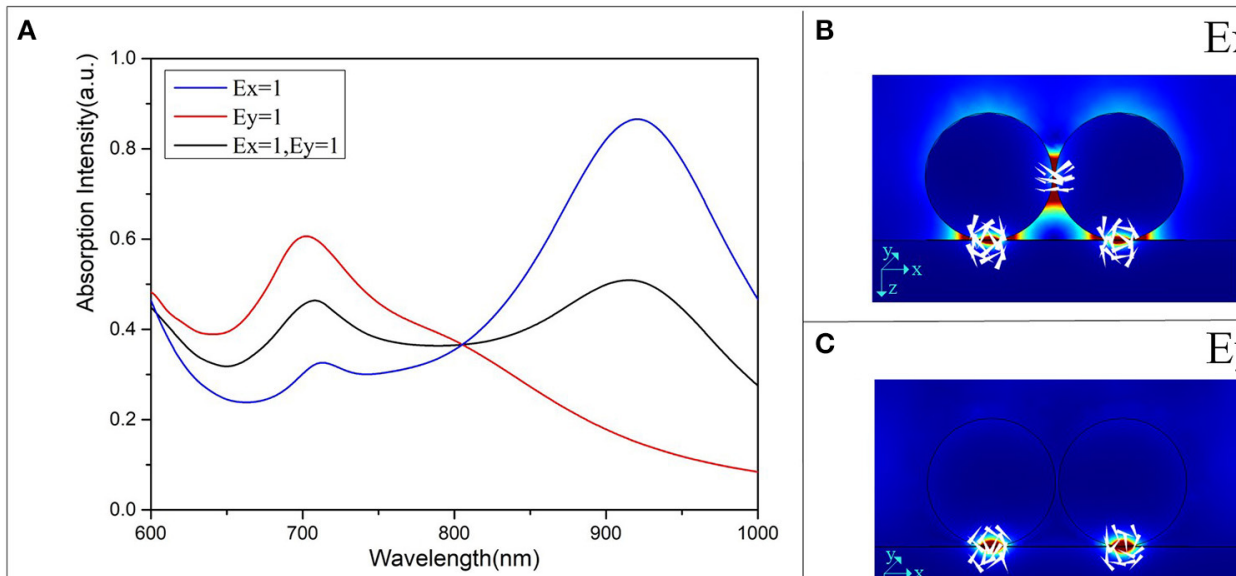

$\mathrm{Ex}=1$
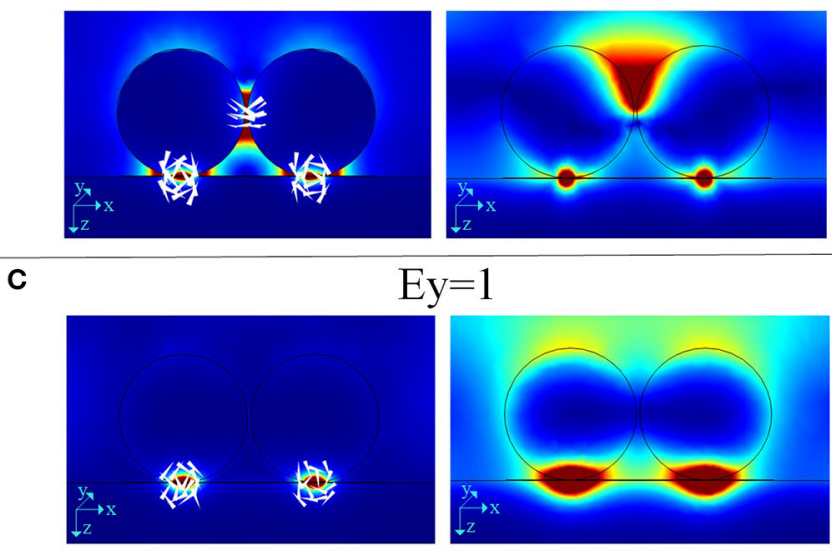

\section{(}
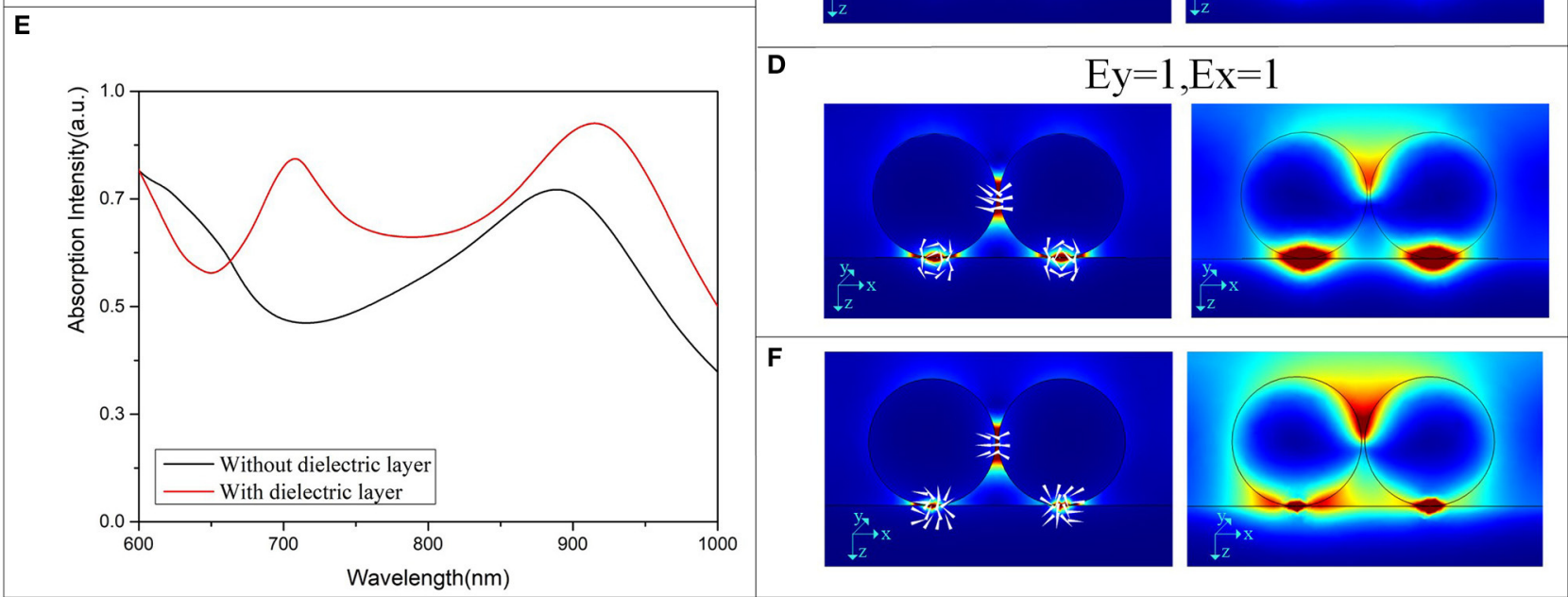

FIGURE 4 | (A) Absorption spectra of Au nanosphere dimer-dielectric-gold film structure with different polarization directions. (B-D) Distribution of the electric field (left) and the magnetic field (right) located at $710 \mathrm{~nm}$ corresponding to Ex $=1$, Ey =1, and Ex $=$ Ey $=1$. (E) The absorption spectra of the structure with and without the dielectric layer. (F) Distribution of the electric field (left) and the magnetic field (right) located at 710 nm corresponding to the structure without the dielectric layer. 
hotspots that are located between the nanosphere with radius $R_{1}$ and the nanosphere with radius $R_{2}\left(\mathrm{AuNP}_{1}-\mathrm{AuNP}_{2}\right)$, between the nanosphere with radius $R_{1}$ and the $\mathrm{Au}$ film ( $\mathrm{AuNP}_{1}$-Film), and between the nanosphere with radius $R_{2}$ and the $\mathrm{Au}$ film $($ AuNP 2 -Film). There was an insignificant change in the intensity at the hotspot of $\mathrm{AuNP}_{1}-\mathrm{AuNP}_{2}$ with a decrease in $t_{1}$, and the hotspot of AuNP ${ }_{1}$-Film decreased, while the hotspot of $\mathrm{AuNP}_{2}$ Film reached maximum when $t_{1}$ was $1 \mathrm{~nm}$.

\section{Effects of the Polarization Direction}

Considering the mutual coupling between the two nanospheres, the optical properties and the near-field enhancement effect are also studied when the polarization direction of the incident light is changed. Figure 4A shows the absorption spectra of the $\mathrm{Au}$ nanosphere dimer-dielectric-Au film structure under the incident light with different polarization directions. It can be seen that the polarization direction has little effect on the peak around $700 \mathrm{~nm}$, whereas the peak around $900 \mathrm{~nm}$ strongly depends on the electric field polarization in the $x$ direction. In other words, as long as the electric field has a component along the $x$ direction where the two spheres contact, the dipole mode between the two nanospheres can be excited. It is also confirmed that the absorption peak around $900 \mathrm{~nm}$ originated from the electric dipole between the two nanospheres. Figures 4B-D shows the distribution of the electric field (left) and the magnetic field (right) corresponding to the absorption peak near $700 \mathrm{~nm}$ for the incident light with different polarization directions. A clear current loop and localized magnetic hot spots are generated between the Au nanospheres and the $\mathrm{Au}$ film around $700 \mathrm{~nm}$, suggesting that the magnetic mode is almost unaffected by the polarization direction of the electric field.

To further investigate the effect of the dielectric layer in exciting the magnetic mode, the absorption spectrum of the structure without the dielectric layer was calculated, as shown in Figure 4E. Compared to the structure with the dielectric layer,

\section{REFERENCES}

1. Sipova-Jungova H, Jurgova L, Mrkvova K, Lynn NS, Spackova B, Homola J. Biomolecular charges influence the response of surface plasmon resonance biosensors through electronic and ionic mechanisms. Biosens Bioelectron. (2019) 126:365-72. doi: 10.1016/j.bios.2018.11.002

2. Zhang X, Liu F, Yan X, Liang L, Wei D. Symmetric and antisymmetric multipole electric-magnetic fano resonances in elliptic disknonconcentric split ring plasmonic nanostructures. J Optics. (2020) 22. doi: 10.1088/2040-8986/abbf8b

3. Tiguntseva EY, Baranov DG, Pushkarev AP, Munkhbat B, Komissarenko F, Franckevicius $\mathrm{M}$, et al. Tunable hybrid fano resonances in Halide perovskite nanoparticles. Nano Lett. (2018) 18:5522-9. doi: 10.1021/acs.nanolett.8b01912

4. Luk'yanchuk B, Zheludev NI, Maier SA, Halas NJ, Nordlander P, Giessen H, et al. The fano resonance in plasmonic nanostructures and metamaterials. Nat Mater. (2010) 9:707-15. doi: 10.1038/nmat2810

5. Nazir A, Panaro S, Proietti Zaccaria R, Liberale C, De Angelis F, Toma A. fano coil-type resonance for magnetic hot-spot generation. Nano Lett. (2014) 14:3166-71. doi: 10.1021/nl500452p

6. Limonov MF, Rybin MV, Poddubny AN, Kivshar YS. Fano resonances in photonics. Nat Photonics. (2017) 11:543-54. doi: 10.1038/nphoton.2017.142 the absorption peak of the one without dielectric layer at around $700 \mathrm{~nm}$ disappeared and its corresponding displacement current loop is non-existent (Figure 4F), which further proved the vital role of the dielectric layer to excite the magnetic mode of the structure [24].

\section{CONCLUSIONS}

Our study proposes an $\mathrm{Au}$ nanosphere dimer-dielectric-gold film sandwiched structure that generates magnetic field-based Fano resonance from visible to near-infrared wavelength. By changing the size of the gold nanosphere and the thickness of the dielectric layer, the Fano resonance wavelength and the resonance intensity can be easily tuned. The proposed structure is also sensitive to the incident polarization direction. Owing to its flexible tuning, high absorption, and low loss characteristics, this structure has a great potential in applications such as resonators, surface-enhanced Raman scattering, and nano-antennas.

\section{DATA AVAILABILITY STATEMENT}

The raw data supporting the conclusions of this article will be made available by the authors, without undue reservation.

\section{AUTHOR CONTRIBUTIONS}

FG and ZW modeled the problem. TG, FG, and WH explored the solution and carried out numerical computations. FG and $\mathrm{XZ}$ analyzed the finding of the study. All authors reviewed the manuscript.

\section{FUNDING}

The authors acknowledge the financial support from the National Natural Science Foundation of China (61905035).

7. Cao G, Dong S, Zhou LM, Zhang Q, Deng Y, Wang C, et al. Fano resonance in artificial photonic molecules. Adv Optical Mater. (2020) 8:1902153. doi: 10.1002/adom.201902153

8. Pena-Rodriguez O, Diaz-Nunez P, Gonzalez-Rubio G, Manzaneda-Gonzalez V, Rivera A, Perlado JM, et al. Au@Ag core-shell nanorods support plasmonic fano resonances. Sci Rep. (2020) 10:5921. doi: 10.1038/s41598-020-62852-9

9. Lassiter JB, Sobhani H, Fan JA, Kundu J, Capasso F, Nordlander P, et al. fano resonances in plasmonic nanoclusters: geometrical and chemical tunability. Nano Lett. (2010) 10:3184-9. doi: 10.1021/nl102108u

10. Ding J, Arigong B, Ren H, Shao J, Zhou M, Lin Y, et al. Dynamically tunable fano metamaterials through the coupling of graphene grating and square closed ring resonator. Plasmonics. (2015) 10:1833-9. doi: 10.1007/s11468-015-0003-6

11. Zhou C, Huo Y, Nini C, Jiang X, Guo Y, Hou Y, et al. Generation and manipulation of the fano resonance with high refractive index sensitivity based on the square split ring dimer. Mater Res Exp. (2019) 6:105064. doi: 10.1088/2053-1591/ab3b85

12. Bazgir M, Zarrabi FB. A switchable split ring resonator nanoantenna design with organic material composite as a refractive index sensor. Optics Commun. (2020) 475:126211. doi: 10.1016/j.optcom.2020. 126211 
13. Modi KS, Kaur J, Singh SP, Tiwari U, Sinha RK. Extremely high figure of merit in all-dielectric split asymmetric arc metasurface for refractive index sensing. Optics Commun. (2020) 462:125327. doi: 10.1016/j.optcom.2020.125327

14. Moreau A, Ciraci C, Mock JJ, Hill RT, Wang Q, Wiley BJ, et al. Controlledreflectance surfaces with film-coupled colloidal nanoantennas. Nature. (2012) 492:86-9. doi: 10.1038/nature11615

15. Ding S-Y, Yi J, Li J-F, Ren B, Wu DY, Panneerselvam R, et al. Nanostructurebased plasmon-enhanced Raman spectroscopy for surface analysis of materials. Nat Rev Mater. (2016) 1:1-16. doi: 10.1038/natrevmats.2016.36

16. Kurouski D, Large N, Chiang N, Henry A-I, Seideman T, Schatz GC, et al. Unraveling the near- and far-field relationship of $2 \mathrm{~d}$ surfaceenhanced Raman spectroscopy substrates using wavelength-scan surfaceenhanced Raman excitation spectroscopy. J Phys Chem C. (2017) 121:1473744. doi: 10.1021/acs.jpcc.7b04787

17. Qin F, Lai Y, Yang J, Cui X, Ma H, Wang J, et al. Deep fano resonance with strong polarization dependence in gold nanoplate-nanosphere heterodimers. Nanoscale. (2017) 9:13222-34. doi: 10.1039/C7NR04524G

18. Ai B, Song C, Bradley L, Zhao Y. Strong fano resonance excited in an array of nanoparticle-in-ring nanostructures for dual plasmonic sensor applications. J Phys Chem C. (2018) 122:20935-44. doi: 10.1021/acs.jpcc.8b05154

19. Dutta A, Alam K, Nuutinen T, Hulkko E, Karvinen P, Kuittinen M, et al. Influence of fano resonance on SERS enhancement in Fano-plasmonic oligomers. Opt Express. (2019) 27:30031-43. doi: 10.1364/OE.27.030031

20. Li J, Deng TS, Liu X, Dolan JA, Scherer NF, Nealey PF. Hierarchical assembly of plasmonic nanoparticle heterodimer arrays with tunable Sub-5 nm nanogaps. Nano Lett. (2019) 19:4314-20. doi: 10.1021/acs.nanolett.9b00792

21. Luo X, Xing Y, Galvan DD, Zheng E, Wu P, Cai C, et al. Plasmonic gold nanohole array for surface-enhanced Raman scattering detection of DNA methylation. ACS Sens. (2019) 4:1534-42. doi: 10.1021/acssensors.9b00008

22. Mahmoudpour M, Ezzati Nazhad Dolatabadi J, Torbati M, Homayouni-Rad A. Nanomaterials based surface plasmon resonance signal enhancement for detection of environmental pollutions. Biosens Bioelectron. (2019) 127:7284. doi: 10.1016/j.bios.2018.12.023

23. Chow TH, Lai Y, Lu W, Li N, Wang J. Substrate-Enabled Plasmonic Color Switching with Colloidal Gold Nanorings. ACS Mater Lett. (2020) 2:74453. doi: 10.1021/acsmaterialslett.0c00182

24. Cui X, Qin F, Lai Y, Wang H, Shao L, Chen H, et al. Molecular tunnel junctioncontrolled high-order charge transfer plasmon and fano resonances. ACS Nano. (2018) 12:12541-50. doi: 10.1021/acsnano.8b07066

25. Du K, Li P, Gao K, Wang H, Yang Z, Zhang W, et al. Strong coupling between dark plasmon and anapole modes. J Phys Chem Lett. (2019) 10:4699705. doi: 10.1021/acs.jpclett.9b01844
26. Chen Z, Zhang S, Chen Y, Liu Y, Li P, Wang Z, et al. Double fano resonances in hybrid disk/rod artificial plasmonic molecules based on dipolequadrupole coupling. Nanoscale. (2020) 12:9776-85. doi: 10.1039/D0NR $00461 \mathrm{H}$

27. Shafiei F, Monticone F, Le KQ, Liu XX, Hartsfield T, Alu A, et al. A subwavelength plasmonic metamolecule exhibiting magnetic-based optical fano resonance. Nat Nanotechnol. (2013) 8:95-9. doi: 10.1038/nnano. 2012.249

28. Liu K, Xue X, Sukhotskiy V, Furlani EP. Optical fano resonance in selfassembled magnetic-plasmonic nanostructures. J Phys Chem C. (2016) 120:27555-61. doi: 10.1021/acs.jpcc.6b09473

29. Jiang X, Huo Y, Zhou C, Guo Y, Hou Y, Niu Q. High-order magnetic modes and multiple Fano resonances generation by a fish-like dimer nanostructure. Phys Scrip. (2020) 95:055507. doi: 10.1088/1402-4896/ab7850

30. Tang C, Yan Z, Wang Q, Chen J, Zhu M, Liu B, et al. Ultrathin amorphous silicon thin-film solar cells by magnetic plasmonic metamaterial absorbers. RSC Adv. (2015) 5:81866-74. doi: 10.1039/C5RA15177E

31. Chen S, Zhang Y, Shih TM, Yang W, Hu S, Hu X, et al. Plasmoninduced magnetic resonance enhanced Raman spectroscopy. Nano Lett. (2018) 18:2209-16. doi: 10.1021/acs.nanolett.7b04385

32. Ni X, Wong ZJ, Mrejen M, Wang Y, Zhang X. An ultrathin invisibility skin cloak for visible light. Science. (2015) 349:13104. doi: 10.1126/science.aac9411

33. Liu B, Tang C, Chen J, Zhu M, Pei M, Zhu X. Electrically tunable fano resonance from the coupling between interband transition in monolayer graphene and magnetic dipole in metamaterials. Sci Rep. (2017) 7:17117. doi: 10.1038/s41598-017-17394-y

34. Dregely D, Hentschel M, Giessen H. Excitation and tuning of higher-order fano resonances in plasmonic oligomer clusters. ACS Nano. (2011) 5:820211 doi: $10.1021 / \mathrm{nn} 202876 \mathrm{k}$

Conflict of Interest: The authors declare that the research was conducted in the absence of any commercial or financial relationships that could be construed as a potential conflict of interest.

Copyright (c) 2021 Gong, Guan, Wei, Huang and Zhang. This is an open-access article distributed under the terms of the Creative Commons Attribution License (CC $B Y)$. The use, distribution or reproduction in other forums is permitted, provided the original author(s) and the copyright owner(s) are credited and that the original publication in this journal is cited, in accordance with accepted academic practice. No use, distribution or reproduction is permitted which does not comply with these terms. 